\title{
Procesamiento de Arvejas (Pisum sativum L.). Parte 2: Cinética de Absorción de Agua en Semillas de Arveja, variedad San Isidro y Sureña
}

\author{
Andrés F. Cerón ${ }^{(1)}$, Oswaldo Osorio(1) y Luis F. Garcés ${ }^{(2)}$ \\ (1) Universidad de Nariño, Facultad de Ingeniería Agroindustrial, Grupo de Apoyo a la investigación y \\ Desarrollo Agroalimentario (GAIDA), Ciudad Universitaria Torobajo, Calle $18 \mathrm{Cr} 50$, San Juan de Pasto - \\ Colombia (e-mail: andre5505@hotmail.com; osorio_oswaldo@hotmail.com) \\ (2) Corporación Universitaria Lasallista, Facultad de Ingeniería de Alimentos, Carrera 51 \# 118 Sur - 57, \\ Caldas, Antioquia - Colombia (e-mail: lugarces@lasallista.edu.co)
}

Recibido Ago. 25, 2015; Aceptado Oct. 29, 2015; Versión final Nov. 7, 2015, Publicado Feb. 2016

\section{Resumen}

Se determinó la cinética de absorción de agua en semillas de arveja (Pisum sativum L.) de las variedades San Isidro y Sureña. La rehidratación se realizó a tres temperaturas $\left(6,18\right.$ y $\left.30^{\circ} \mathrm{C}\right)$, empleando una relación semillas/agua $1: 4(\mathrm{p} / \mathrm{v})$, y se tomaron mediciones a $0,3,6,9,12$ y 24 horas. El efecto de la temperatura fue evaluado mediante la capacidad ge adsorción de agua, mientras que la cinética de adsorción de agua fue estudiada con dos modelos (Exponencial y Peleg!s). La capacidad de adsorción de agua aumentó con el incremento de la temperatura del agua. Los modelos expuestos describen adecuadamente la cinética de adsorción de agua. Tanto la difusividad como los parámetros cinéticos presentan dependencia con la temperatura y al evaluarlos con la ecuación de Arrhenius se obtienen energías de activación de $17.24 \mathrm{~kJ} / \mathrm{mol}$ para San Isidro y $9.25 \mathrm{~kJ} / \mathrm{mol}$ para Sureña.

Palabras clave: rehidratación; temperatura; constantes cinéticas; difusividad

\section{Processing of Peas (Pisum sativum L.). Part 2: Kinetics of Absorption of Water in Pea Seeds, varieties San Isidro and Sureña}

\begin{abstract}
Water absorption kinetics in pea seeds (Pisum sativum L.), varieties San Isidro and Sureña, were determined. Rehydration was conducted at three temperatures $\left(6,18\right.$ and $\left.30^{\circ} \mathrm{C}\right)$, using a seed/water ratio 1: $4(\mathrm{w} / \mathrm{v})$, and measurements at $0,3,6,9,12$ and 24 hours were taken. The effect of temperature was evaluated by the water adsorption capacity, whereas the water adsorption kinetics was studied in two models (Exponential and Peleg). The water adsorption capacity increased with increasing water temperature. The models adequately describe the kinetics of adsorption of water. Both diffusivity and kinetic parameters show dependence on temperature and when are evaluated by the Arrhenius activation energy of $17.24 \mathrm{~kJ} / \mathrm{mol}$ for San Isidro and $9.25 \mathrm{~kJ} / \mathrm{mol}$ for Sureña were obtained.
\end{abstract}




\section{INTRODUCCIÓN}

La arveja (Pisum sativum L.) es uno de los principales cultivos comestibles en el mundo (Amarakoon et al., 2012), ocupa el cuarto lugar en la producción mundial junto a la soja, maní y frijol (Villalobos et al., 2013), es fuente de proteína con aminoácidos esenciales, hidratos de carbono complejos, fibra dietética y microelementos: fósforo, calcio, magnesio, hierro y vitaminas: C, B1, B2, PP, E y carotenos (De Almeida et al., 2006; Szymanowska et al., 2009; Wang et al., 2011). El consumo de esta leguminosa, se da en estado fresco o seco $(<14 \%$ humedad base húmeda), siendo la ventaja de este último, la conservación del alimento por mayor tiempo, la reducción de la humedad previene el crecimiento de microorganismos y minimiza las reacciones de deterioro (Ibarz y Barbosa-Canovas, 2005; Téllez-Pérez et al., 2012). Sin embargo, los alimentos en este estado, deben ser rehidratados para su consumo o uso agroindustrial (Parthasarathi y Anandharamakrishna, 2014), esta es una operación compleja que ayuda a restaurar las propiedades del alimento fresco, anteriormente deshidratado (Brousse et al., 2012); no obstante, aún son escasas las investigaciones referentes al fenómeno de absorción de agua por parte de las semillas de arveja. Turhan et al., (2002) y Yildirim et al., (2011) mencionan que la comprensión de la absorción de agua en las legumbres es de importancia ya que afecta a los procesos posteriores. Tal como lo cita Cerón et al., (2015) en el proceso de elaboración de arveja rehidratada en conserva (envasado y esterilización). Así mismo, Sharma et al., (2011) resalta que la construcción de equipos de clasificación, separación, transporte, entre otros, se diseñan en función del contenido de agua.

En este sentido, Paksoy y Aydin (2006), Yalcin et al., (2007) y Wang et al., (2008) realizaron la rehidratación de las semillas de arveja (Pisum sativum L.) estudiando el efecto de la variedad y el proceso sobre los nutrientes y los cambios en las propiedades físicas de las semillas con forme aumento el contenido de humedad, pese a lo anterior no se estudió el proceso de adsorción de agua expresado mediante un modelo matemático. Actualmente, este fenómeno se ha estudiado bajo la primera y segunda ley de Fick (Yildirim et al., 2011) y bajo la utilización de modelos empírico, para la predicción y la optimización de la cinética de rehidratación; Sopade et al., (2007) concluyeron que los modelos de Pilosof, Singh y Kulshrestha y Peleg, son matemáticamente equivalentes y puede usarse cualquiera de ellos para modelar este fenómeno; en el mismo sentido Brousse et al., (2012) y Brousse et al., (2014) concluyeron que el modelo exponencial es equivalente al modelo de Pilosof, siendo una alternativa para el cálculo de los parámetros cinéticos. En este contexto, el objetivo de esta investigación fue realizar la cinética de absorción de agua en semillas de arveja (Pisum sativum L.) de dos variedades San Isidro y Sureña, determinando y comparando algunos parámetros cinéticos.

\section{MATERIALES Y MÉTODOS}

\section{Material de estudio}

Se utilizaron semillas de arvejas (Pisum sativum L.) de la variedad San Isidro (semilla verde, hilum blanco, lisa) y Sureña (semilla verde, hilum negro, lisa), el material se recolectó al sexto mes de establecido el cultivo en la granja del Centro Internacional de Producción Limpia LOPE, Sena Regional Nariño, ubicado a 2650 metros sobre el nivel del mar, temperatura promedio $13 \stackrel{\circ}{\circ}$ y precipitación promedio anual de $700 \mathrm{~mm}$, condiciones favorables para el cultivo. Los materiales fueron inspeccionados de acuerdo a la norma técnica colombiana NTC 791 solo se utilizó semillas con diámetro promedio mayor a $6.35 \mathrm{~mm}$; la humedad promedio de las semillas se encontró entre 16 y 17 \% (base húmeda).

\section{Rehidratación del material}

La humedad de los materiales se llevó hasta un $14.211 \pm 1.184 \%$ (base húmeda) para la variedad San Isidro y $12.911 \pm 1.924 \%$ (base húmeda) para la variedad Sureña, empleando un secador de bandejas construido por Industrias Químicas FIQ LTDA, a una temperatura de $45{ }^{\circ} \mathrm{C}$ con velocidad de aire de $2 \mathrm{~m} / \mathrm{s}$ durante 6 horas; posteriormente se determinó la esfericidad de las semillas para ello se midieron las tres dimensiones lineales (longitud (L), anchura (W) y grueso (T), utilizando un calibrador con precisión de 0.05 milímetros; posteriormente se aplicó la ecuación 1 (Yalcin et al., 2007); así mismo, se realizó la caracterización químico proximal de los materiales según el método de análisis de alimentos propuesto por Bernal (1998) determinando: energía, humedad, materia seca, ceniza, extracto etéreo, fibra cruda, proteína, extracto no nitrogenado, calcio, hierro.

Caracterizado el material, se realizó la rehidratación por inmersión en recipientes de polietileno de alta densidad (capacidad $3 \mathrm{~L}$ ), utilizando agua destilada en relación semillas/agua 1:4 (p/v), por espacio de 24 horas, tomando mediciones a las $0,3,6,9,12$ y 24 horas a tres diferentes temperaturas $\left(6^{\circ} \mathrm{C}\right.$, temperatura ambiente $18{ }^{\circ} \mathrm{C}$ y $30^{\circ} \mathrm{C}$ ); para el control de la temperatura se utilizó un baño termostático Eyela OSB 2000, con precisión de $\pm 0.1^{\circ} \mathrm{C}$ y un equipo de enfriamiento Chiller Polyscience SD28R,con precisión $\pm 0.1^{\circ} \mathrm{C}$; el registro de la temperatura se realizó utilizando un Datalogger Oakton Temp 10T. 


$$
\emptyset=\frac{(\mathrm{LWT})^{\frac{1}{3}}}{\mathrm{~L}}
$$

\section{Determinación humedad}

Se eliminó la humedad superficial de las semillas mediante el uso de toallas de papel; se utilizó la metodología descrita por Bernal (1998), empleando un horno eléctrico (Thermolab Dies) a $100{ }^{\circ} \mathrm{C}$, por un tiempo de 24 horas hasta lograr un peso constante; la humedad se expresó en $\mathrm{g}$ de agua / $\mathrm{g}$ solido seco.

Capacidad de absorción de agua (RC)

Se determinó mediante la metodología expuesta por Melquíades et al., (2009) empleando la ecuación 2.

$$
R C=\frac{W r}{W d}
$$

Donde: Wr: peso total al final del proceso de rehidratación y Wd: peso del material seco.

\section{Determinación constante de velocidad de rehidratación}

Las constantes cinéticas de velocidad de rehidratación se determinaron mediante la metodología descrita por Brousse et al., (2012), modelo exponencial (ecuación 3) y la metodología descrita por Jideani y Mpotokwana (2009) modelo de Peleg!s (ecuación 4).

$$
\begin{aligned}
& C_{(t)}=C_{\infty}\left(1-\exp ^{(-k t)}\right) \\
& C_{(t)}=C_{0}+\frac{t}{K_{1}+K_{2} t}
\end{aligned}
$$

Donde: $C_{(t)}$ : contenido de agua a tiempo $t, g$ de agua/g de sólido seco; $C_{\infty}$ : contenido de agua en el equilibrio a tiempo infinito, g de agua/g de solido seco; Co: contenido de humedad inicial, g de agua/g de sólido seco; K: constante de velocidad de absorción de agua, $\min ^{-1}$; t: tiempo de adsorción de agua (min o h). $\mathrm{K}_{1}$ : constante de velocidad de Peleg $\left(\mathrm{h}^{-1}{ }^{-1}\right) ; \mathrm{K}_{2}$ : constante de capacidad de Peleg $\left(\%^{-1}\right)$.

Los parámetros cinéticos $\mathrm{C}_{\infty}, \mathrm{K}, \mathrm{K}_{1}$ y $\mathrm{K}_{2}$ se obtuvieron a partir de los datos experimentales mediante regresión no lineal.

\section{Estudio de la transferencia de agua como modelo difusivo}

Se utilizó la metodología descrita por Melquíades et al., (2009) a partir de la segunda Ley de Fick, ecuación de difusión, con geometría de un cilindro (ecuación 5).

$$
\frac{\mathrm{m}-\mathrm{ms}}{\mathrm{mo}-\mathrm{ms}}=\frac{4}{5.783} \exp \left(\frac{5.783 \text { Def } \mathrm{t}}{\mathrm{R}^{2}}\right)
$$

Donde: Def: coeficiente de difusión de agua en el grano; t: tiempo; mo: humedad inicial; ms: humedad en equilibrio y $\mathrm{R}$ : radio de la semilla equivalente.

En el mismo sentido, se determinó la energía de activación (Ea), de la operación de rehidratación, a partir del coeficiente de difusión que se ajusta a la ecuación de Arrhenius debido a su dependencia con la temperatura (Melquíades et al., 2009) ecuación 6.

$$
\text { Def=Do exp }{ }^{\left(-\frac{E a}{R T}\right)}
$$

Donde: $D_{0}$ : factor pre-exponencial; Ea: energía de activación $(\mathrm{kJ} / \mathrm{mol})$ y $\mathrm{R}$ : constante de los gases $(8.314 \mathrm{~J} /$ (g mol K).

\section{Análisis estadístico}

Todos los resultados se expresaron como media más o menos la desviación estándar. Los gráficos fueron realizados con ayuda del programa Sigma Plot 10 (SPSS, USA); así mismo, se efectuó el análisis de 
varianza y prueba de comparación mediante la mínima diferencia significativa (LSD) de Fisher a un $5 \%$ de nivel de significancia mediante el programa Statgraphics centurión (c) Plus versión XV.II. La bondad de ajuste del modelo se evaluó con los parámetros $R^{2}$ (coeficiente de determinación), \% $\mathrm{E}$ (error porcentual promedio) ecuación 7 y RMSE (raíz cuadrada del error medio cuadrático) ecuación 8.

$$
\begin{gathered}
\% \mathrm{E}=\frac{\sum_{\mathrm{i}=1}^{\mathrm{n}} \frac{\mid \text { Ccal-Cexp } \mid \mathrm{n}}{\text { Cexp } n}}{\mathrm{n}} \cdot 100 \\
\text { RMSE }=\left[\frac{\sum_{\mathrm{i}=1}^{\mathrm{n}}(\text { Ccal-Cexp })^{2}}{\mathrm{n}}\right]^{0.5}
\end{gathered}
$$

Donde: Ccal: valor calculado; Cexp: valor experimental; $n$ : número de determinaciones. Varios autores citados por Brousse et al., (2014) mencionan que un buen ajuste es indicado por $R^{2}>85$, E \% < $10 \%$ y valores pequeños de RMSE indicando que los valores del pronóstico son muy aproximados a los valores reales, valores grandes significan que existen grandes errores en el pronóstico. En este estudio se aceptan RSME $<0.3$.

\section{RESULTADOS Y DISCUSION}

\section{Análisis físico y proximal}

La variedad San Isidro presentó valores de esfericidad de $0.909 \pm 0.022$ mientras que la variedad Sureña $0.890 \pm 0.033$; siendo estos valores cercanos a los valores reportados para geometría de un cilindro 0.874 y distantes de los valores de una esfera 1.0 y un cubo 0.804 (Bayram, 2005). Respecto del análisis proximal los resultados se expresaron en base húmeda, para cada variedad, en la tabla 1 se muestran los valores.

Tabla 1: Composición proximal semillas de arveja (Pisum sativum L.)

\begin{tabular}{|l|c|c|c|}
\hline \multirow{2}{*}{ Característica } & \multirow{2}{*}{ Unidades } & \multicolumn{2}{|c|}{ Variedad } \\
\cline { 3 - 4 } & & San Isidro & Sureña \\
\hline Energía & $\mathrm{Kcal} / 100 \mathrm{~g}$ & 367.767 & 375.428 \\
\hline Humedad & $\mathrm{g} / 100 \mathrm{~g}$ & 14.322 & 12.911 \\
\hline Materia seca & $\mathrm{g} / 100 \mathrm{~g}$ & 85.678 & 87.089 \\
\hline Extracto etéreo & $\mathrm{g} / 100 \mathrm{~g}$ & 1.271 & 1.397 \\
\hline Proteína & $\mathrm{g} / 100 \mathrm{~g}$ & 20.180 & 19.863 \\
\hline Carbohidratos totales & $\mathrm{g} / 100 \mathrm{~g}$ & 56.856 & 57.749 \\
\hline Fibra cruda & $\mathrm{g} / 100 \mathrm{~g}$ & 4.393 & 5.519 \\
\hline Ceniza & $\mathrm{g} / 100 \mathrm{~g}$ & 2.627 & 2.678 \\
\hline Calcio & $\mathrm{mg} / 100 \mathrm{~g}$ & 61.871 & 63.593 \\
\hline Hierro & $\mathrm{mg} / 100 \mathrm{~g}$ & 4.911 & 5.006 \\
\hline
\end{tabular}

Capacidad de absorción de agua $(R C)$

El análisis de varianza (ANOVA) solo mostró influencia significativa de la temperatura sobre el RC de la variedad San Isidro $(p<0.05)$. Respecto de la comparación entre variedades, no se encontró diferencias en cada una de las temperatura objeto de estudio ( $p>0.05$ ). Sin embargo, se evidenció aumentos de los RC, debido a que la absorción de agua se facilitada a mayores temperaturas (Bello et al., 2007; Salimi et al., 2011; Muñoz et al., 2012). Este fenómeno puede estar relacionado con la alta tasa de difusión del agua y solutos a mayor temperatura, otorgando una reducción importante del tiempo de rehidratación (Marín et al., 2006; Kashiri et al., 2010). Los valores promedios de RC en función de la temperatura, se muestran en la tabla 2.

Según la tabla 2 los valores de RC de la variedad San Isidro fueron mayores que la variedad Sureña, una posible explicación de este fenómeno, sería el mayor grado de deshidratación que sufrió dicha variedad durante el secado, Salimi et al., (2011) mencionan que la operación de rehidratación depende de cambios estructurales en los tejidos o células del material alimentario, que se dan durante el secado, operación que ocasiona la contracción y el colapso de los mismos, reduciendo la capacidad de absorción de agua, explicando el fenómeno observado. 
Tabla 2: Capacidad de absorción de agua (RC), letras no comunes implican diferencias entre promedios, según prueba de LSD de Fisher a un 95\% de confianza).

\begin{tabular}{|c|c|c|}
\hline \multirow{2}{*}{ Temperatura $\left({ }^{\circ} \mathrm{C}\right)$} & \multicolumn{2}{|c|}{ RC (g semilla rehidratada / g semilla seca) } \\
\cline { 2 - 3 } & San Isidro & Sureña \\
\hline 6 & $1.831 \pm 0.104^{\mathrm{a}}$ & $1.835 \pm 0.121^{\mathrm{a}}$ \\
\hline 18 & $1.979 \pm 0.119^{\mathrm{b}}$ & $1.928 \pm 0.126^{\mathrm{ab}}$ \\
\hline 30 & $1.997 \pm 0.060^{\mathrm{b}}$ & $1.965 \pm 0.102^{\mathrm{b}}$ \\
\hline
\end{tabular}

\section{Cinética de adsorción de agua}

En la figura 1 se muestra el comportamiento cinético de la rehidratación de las semillas de arveja (Pisum sativum L.) de las dos variedades. La velocidad de absorción de agua fue mayor al inicio de la operación de rehidratación, para luego disminuir lentamente hasta el valor de equilibrio, Lucas et al., (2007) observaron igual fenómeno en la rehidratación de cereales para desayuno (crispís de arroz), Cunningham et al., (2008) en la rehidratación de papa, Yildirim et al., (2010) y Shafaei y Masoumi (2014a) en la rehidratación de garbanzos. Este fenómeno se debe a que todos los espacios intercelulares 0 intracelulares quedan saturados con agua conforme transcurre el tiempo (Marín et al., 2006).
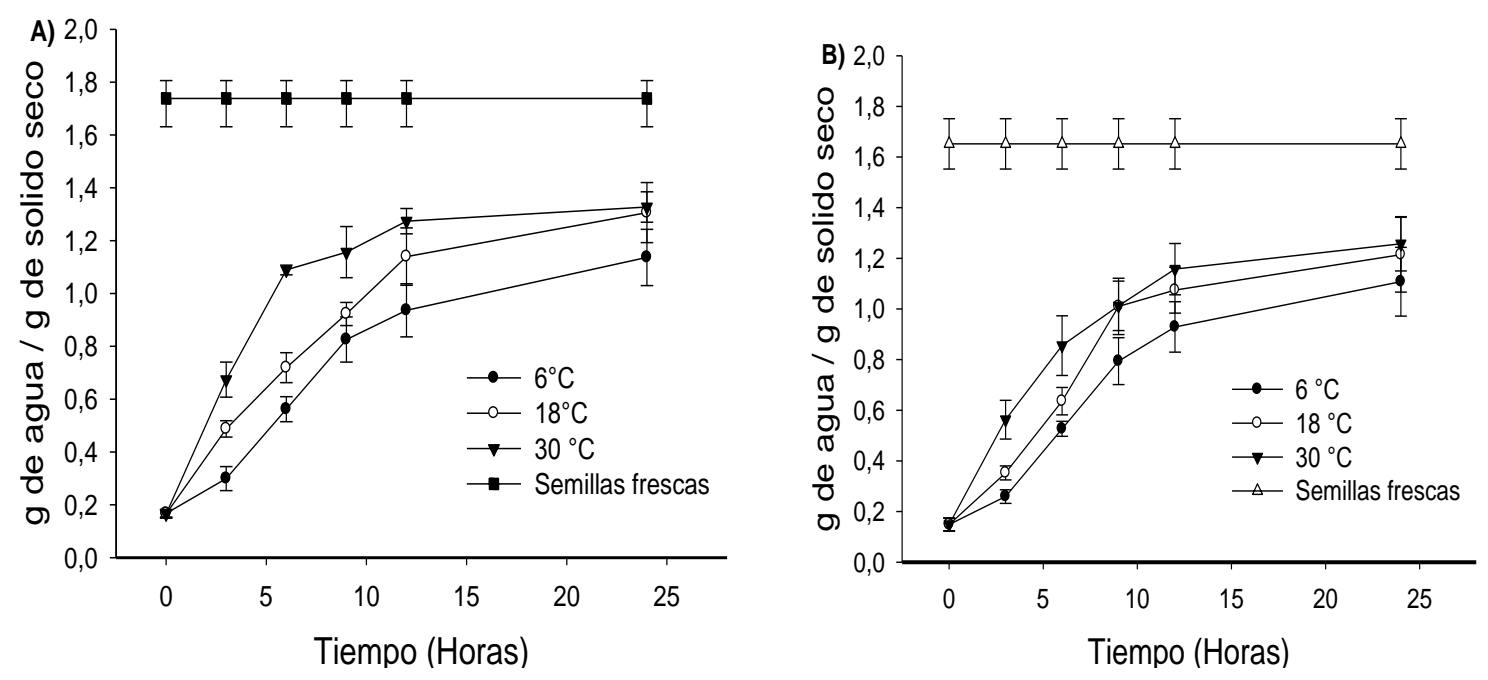

Fig. 1: Cinética de rehidratación semillas de arveja: A) San Isidro, B) Sureña.

El ajuste de los datos experimentales mostró que el modelo exponencial (tablas 3) describe mejor la absorción de agua en las semillas de arveja de las dos variedades, como lo muestran los valores de $R^{2}$, RMSE y E \%; no obstante, el modelo de Peleg!s (tabla 4) es una buena opción para describir la absorción de agua de la variedad San Isidro a temperaturas de 18 y $30^{\circ} \mathrm{C}$, y para la variedad Sureña a temperatura de $30{ }^{\circ} \mathrm{C}$. Parthasarathi y Anandharamakrishna (2014) afirman que varios autores en sus estudios concluyeron que el modelo de Peleg describe adecuadamente la hidratación de diversos productos alimenticios, confirmando lo obtenido.

Así mismo, los datos muestran que a menor temperatura, la humedad en equilibrio alcanzada fue menor; resultados similares reportaron Melquíades et al., (2009) durante la rehidratación de zanahoria. Pese a lo anterior, no se evidenció diferencia significativas entre las humedades en equilibrio alcanzadas a las 24 horas de rehidratación, tanto de la semillas de variedad San Isidro como de la variedad Sureña ( $p>0.05)$; no obstante, existen diferencias significativas entre los valores de humedad de las semillas en estado fresco, frente a los valores obtenidos en el equilibrio $(p<0.05)$, lo cual permite afirmar que los cambios estructurales que sufrieron las semillas durante el secado son irreversibles, Krokida y Philippopoulos (2005) observaron efectos similares en varias frutas y verduras deshidratadas. Con respecto a la comparación entre variedades no se encontró diferencias significativas entre las humedades en equilibrio alcanzadas en cada una de las temperaturas $(p>0.05)$.

Por otra parte, al aumentar la temperatura las constates de velocidad de absorción de agua aumentaron significativamente $(p<0.05)$ para las dos variedades (tabla 3$)$. Mientras que bajo el modelo de Peleg!s (tabla 4) los valores de $K_{1}$ fueron inversos a la temperatura. Jideani y Mpotokwana (2009) reportaron conclusión similar afirmando que el tiempo y la temperatura tienen efecto significativo sobre la velocidad de rehidratación de semillas de bambara; así mismo, Kashiri et al., (2010) mencionan que el efecto inverso observado indica una creciente velocidad de absorción de agua a temperaturas más altas. 
Tabla 3: Parámetros cinéticos y bondad de ajuste del modelo exponencial. (Letras no comunes implican diferencias entre promedios, según prueba de LSD de Fisher a un 95\% de confianza).

\begin{tabular}{|c|c|c|c|c|c|c|}
\hline \multirow{2}{*}{ Variedad } & \multirow{2}{*}{ Temperatura ${ }^{\circ} \mathrm{C}$} & \multirow{2}{*}{$\begin{array}{l}\mathrm{C}_{(\infty)}(\mathrm{g} \text { de agua/g de } \\
\text { solido seco }\end{array}$} & \multirow{2}{*}{${ }^{*} \mathrm{~K}\left(\mathrm{x} 10^{-3} \mathrm{~min}^{-1}\right)$} & \multirow{2}{*}{$\mathrm{R}^{2}$} & \multicolumn{2}{|c|}{ Bondad de ajuste } \\
\hline & & & & & RMSE & $E \%$ \\
\hline \multirow{3}{*}{ San Isidro } & 6 & $1.136 \pm 0.106^{a}$ & $2.304 \pm 0.121^{a}$ & 96.221 & 0.225 & 10.052 \\
\hline & 18 & $1.130 \pm 0.113^{a}$ & $2.558 \pm 0.113^{a}$ & 95.354 & 0.101 & 4.495 \\
\hline & 30 & $1.327 \pm 0.057^{a}$ & $4.170 \pm 0.152^{b}$ & 97.772 & 0.059 & 2.637 \\
\hline \multirow{3}{*}{ Sureña } & 6 & $1.107 \pm 0.135^{\mathrm{a}}$ & $2.422 \pm 0.112^{\mathrm{a}}$ & 94.998 & 0.334 & 13.931 \\
\hline & 18 & $1.214 \pm 0.147^{a}$ & $3.198 \pm 0.156^{b}$ & 94.260 & 0.261 & 11.155 \\
\hline & 30 & $1.257 \pm 0.107^{a}$ & $3.311 \pm 0.212^{b}$ & 98.228 & 0.111 & 4.973 \\
\hline
\end{tabular}

Respecto de la constante $\mathrm{K}_{2}$, está inversamente relacionada con la capacidad de absorción de agua (Jideani y Mpotokwana, 2009). En la tabla 3 se muestras aumentos significativos de $\mathrm{K}_{2}$ conforme se incrementó la temperatura, Jideani y Mpotokwana (2009) observaron efecto similar en la rehidratación de semillas de bambara, afirmando que lo anterior demuestra que velocidad de absorción de agua se incrementó y la capacidad de absorción de agua disminuyó con el aumento de la temperatura.

Tabla 4: Parámetros cinéticos y bondad de ajuste del modelo de Peleg!s. (Letras no comunes implican diferencias entre promedios, según prueba de LSD de Fisher a un 95\% de confianza).

\begin{tabular}{|c|c|c|c|c|c|c|}
\hline \multirow{2}{*}{ Variedad } & \multirow{2}{*}{ Temperatura $\left({ }^{\circ} \mathrm{C}\right)$} & \multirow{2}{*}{$\begin{array}{c}\mathrm{K}_{1} \\
\left(\times 10^{-2} \mathrm{~h} \%^{-1}\right)\end{array}$} & \multirow{2}{*}{$\begin{array}{c}\mathrm{K}_{2} \\
\left(\times 10^{-2} \mathrm{~h} \%-1\right)\end{array}$} & \multirow{2}{*}{$\mathrm{R}^{2}$} & \multicolumn{2}{|c|}{ Bondad del ajuste } \\
\hline & & & & & RMSE & $E \%$ \\
\hline \multirow{3}{*}{ San Isidro } & 6 & $9.589 \pm 0.532^{\mathrm{a}}$ & $0.515 \pm 0.032^{\mathrm{a}}$ & 89.807 & 0.336 & 15.016 \\
\hline & 18 & $7.077 \pm 0.356^{b}$ & $0.556 \pm 0.026^{a}$ & 96.459 & 0.091 & 4.112 \\
\hline & 30 & $2.699 \pm 0.215^{c}$ & $0.731 \pm 0.028^{b}$ & 98.599 & 0.138 & 6.194 \\
\hline \multirow{3}{*}{ Sureña } & 6 & $10.010 \pm 0.450^{a}$ & $0.593 \pm 0.025^{a}$ & 89.399 & 0.387 & 17.316 \\
\hline & 18 & $6.281 \pm 0.312^{b}$ & $0.649 \pm 0.032^{\mathrm{ab}}$ & 89.699 & 0.371 & 16.606 \\
\hline & 30 & $4.377 \pm 0.413^{c}$ & $0.700 \pm 0.045^{b}$ & 98.732 & 0.087 & 3.911 \\
\hline
\end{tabular}

En la tabla 5 se muestra los coeficientes de difusión, evidenciando la dependencia de este parámetro a medida que aumento la temperatura de rehidratación, confirmado, mediante el análisis de varianza (ANOVA) el cual mostro diferencias estadísticamente significativas entre los valores de difusividad a diferente temperatura para la variedad San Isidro y Sureña $(p<0.05)$. Conclusión similar reportaron Khazaei y Mohammadi (2009) en semillas de sésamo y Shafaei y Masoumi (2014b) en frijol, afirmando que temperaturas más altas aumentan la difusividad y por ende la velocidad de rehidratación, lo cual permite alcanzar la humedad de equilibrio en menor tiempo. Al comparar las dos variedades en cada una de las temperaturas, se determinó que a temperaturas de 6 y $18{ }^{\circ} \mathrm{C}$ San Isidro no presentaron diferencias significativas $(p>0.05)$ respecto de los valores de Sureña a $6{ }^{\circ} \mathrm{C}$. Los valores encontrados se encuentran dentro del rango reportado por varios autores $10^{-9}$ y $10^{-12}$ citados por Téllez-Pérez et al., (2012) y Kashiri et al., (2010).

Tabla 5: Coeficientes de difusión rehidratación de arveja (Pisum sativum L). (Letras no comunes implican diferencias entre promedios, según prueba de LSD de Fisher a un 95\% de confianza).

\begin{tabular}{|c|c|c|c|c|}
\hline \multirow{2}{*}{ Temperatura $\left({ }^{\circ} \mathrm{C}\right)$} & San Isidro & \multirow{2}{*}{$\mathrm{R}^{2}$} & Sureña & \multirow{2}{*}{$\mathrm{R}^{2}$} \\
\cline { 2 - 2 } & Def $\left(\times 10^{-11} \mathrm{~m}^{2} \cdot \mathrm{s}^{-1}\right)$ & & Def $\left(\times 10^{-11} \mathrm{~m}^{2} \cdot \mathrm{s}^{-1}\right)$ & \\
\hline 6 & $6.483 \pm 0.347^{\mathrm{a}}$ & 96.221 & $6.817 \pm 0.336^{\mathrm{a}}$ & 94.991 \\
\hline 18 & $7.197 \pm 0.431^{\mathrm{a}}$ & 95.354 & $9.001 \pm 0.543^{\mathrm{b}}$ & 94.260 \\
\hline 30 & $11.74 \pm 0.612^{\mathrm{b}}$ & 97.714 & $9.318 \pm 0.545^{\mathrm{b}}$ & 98.250 \\
\hline
\end{tabular}




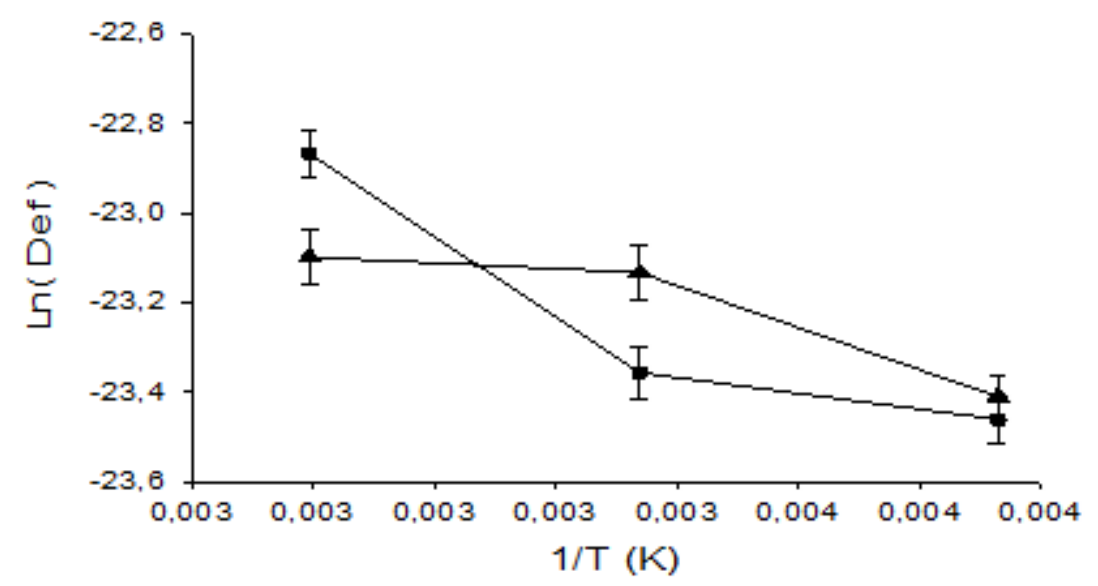

Fig. 2: (匹) San Isidro: $y=-2072.994 x-16.633, R^{2}=0.861$ ( $\mathbf{\Delta}$ ) Sureña: $y=-1113.077 x-19.919, R^{2}=0.849$.

La energía de activación se determinó a partir de la pendiente de la figura 2 (grafico de Arrhenius), los valores obtenidos fueron: San Isidro $17.235 \mathrm{~kJ} / \mathrm{mol}$ y Sureña $9.254 \mathrm{~kJ} / \mathrm{mol}$; según Melquíades et al., (2009) estos valores, termodinámicamente representan la energía que necesitan las moléculas de agua para su migración o movimiento dentro del producto. Los valores de energía de activación obtenidos en esta investigación son razonables al compararse con los datos presentados por diversos autores citados por Kashiri et al., (2010) para otros granos: $24.21 \mathrm{~kJ} / \mathrm{mol}$ para sorgo, 37.62 y $35.69 \mathrm{~kJ} / \mathrm{mol}$ para caupí y bambara y $11.979 \mathrm{~kJ} / \mathrm{mol}$ para trigo.

\section{CONCLUSIONES}

El modelo exponencial utilizado para simular el proceso de rehidratación mostró una buena calidad de ajuste sobre las curvas de rehidratación experimental, validando su utilización; mientras que el modelo de Peleg!s describe adecuadamente la cinética de absorción de agua de la variedad San Isidro a temperaturas de 18 y $30^{\circ} \mathrm{C}$ mientras que para la variedad Sureña a temperatura de $30^{\circ} \mathrm{C}$.

Se determinó influencia estadísticamente significativa de la temperatura sobre la cinética de rehidratación de las semillas de arveja (Pisum sativum L.) tanto para la variedad San Isidro como Sureña, permitiendo inferir que bajo el modelo de Peleg!s el aumento de $\mathrm{K}_{2}$ muestra incremento en la velocidad de absorción de agua mientras que la capacidad de absorción de agua disminuye conforme aumenta la temperatura.

\section{AGRADECIMIENTOS}

Los autores expresan sus agradecimientos a: La Universidad de Nariño; Grupo de Apoyo a la investigación y Desarrollo Agroalimentario (GAIDA), Grupo de investigación Tecnologías Emergentes en Agroindustria (TEA) y al SGR por el apoyo con los proyectos regalías 2012 BPIN 20120001100188.

\section{REFERENCIAS}

Amarakoon, D., D. Thavarajah, K. McPhee, y P. Thavarajah, Iron-, zinc-, and magnesium-rich field peas (Pisum sativum L.) with naturally low phytic acid: A potential food-based solution to global micronutrient malnutrition, doi: org/10.1016/j.jfca.2012.05.007, J Food Compos Anal, 27, 8-13 (2012)

Bayram, M, Determination of the sphericity of granular food materials, doi:10.1016/j.jfca.2012.05.00, J Food Eng, 68, 385-390 (2005)

Bello, M. O., M. P. Tolaba, y C. Suarez, Water absorption and starch gelatinization in whole rice grain during soaking, doi:10.1016/j.Iwt.2005.09.017, LWT- Food Sci Techno, 40, 313-318 (2007)

Bernal De, R. I., Análisis de alimentos. $3^{\text {ra }}$ edición, 58-60. Editora Guadalupe Ltda, Bogotá, Colombia (1998)

Brousse M.M, M. Vergara, E. Zubreski, N. Cruz, y M. Martos. Cinética de absorción de agua de tejidos de mandioca macerados con una poligalacturonasa microbiana,

http://www.scielo.org.ar/scielo.php?script=sci_arttext\&pid=S1851-75872014000200008\&lng=es\&nrm=iso>;

ISSN 1851-7587, Rev Cienc y Tecnol, 16(22), 53-57 (2014) 
Brousse, M. M., A. B. Nieto, A. R. Linares, y M. L. Vergara, Cinética de adsorción de agua en purés deshidratados de mandioca (Manihot esculenta Crantz), http://www.rvcta.org; ISSN: 2218-4384, Rev Venez Cienc y Tecnol Aliment, 3 (1), 80-96 (2012)

Cerón, A. F, L. I. Latorre, M. A. Bucheli, O. Osorio, D. F. Mejia y L. F. Garcés, L. Determinación de constantes de velocidad de rehidratación y cambios sobre algunas propiedades físicas en semillas de arveja (Pisum sativum L.), http://repository.lasallista.edu.co:8080/ojs/index.php/rldi/article/view/786; ISSN: 17944449, Rev Lasallista de Investig, 12(1), 21-32 (2008)

Cunningham, S. E., W. A. Mcminn, T. R. Magee, y P. S. Richardson, Effect of processing conditions on the water absorption and texture kinetics of potato, doi:10.1016/j.jfoodeng.2007.05.007, J Food Eng, 84, 214$223(2008)$

De Almeida, G. E., K. Queiroz-Monici, S. M. Pissini, y A. C. De Oliveira, Chemical composition, dietary fibre and resistant starch contents of raw and cooked pea, common bean, chickpea and lentil legumes, doi:10.1016/j.foodchem.2004.11.020, Food Chem, 94 (3), 327-330 (2006)

Ibarz, A., y G. V. Barbosa-Cánovas, Operaciones unitarias en la ingeniería de alimentos. $1^{\text {ra }}$ edición, 583. Ediciones Mundi-Prensa, Madrid, España (2005)

Jideani, V. A., y S. M. Mpotokwana, Modeling of water absorption of Botswana bambara varieties using Peleg's equation, doi:10.1016/j.jfoodeng.2008.10.040, J Food Eng, 92, 182-188 (2009)

Kashiri, M., M. Kashaninejad, y N. Aghajani, Modeling water absorption of sorghum during soaking, http://www.scielo.org.ar/scielo.php?script=sci_arttext\&pid=S0327-07932010000400014\&lng=es\&nrm=iso>;

ISSN 0327-0793, Lat Am Appl Res, 40, 383-388 (2010)

Khazaei, J., y N. Mohammadi, Effect of temperature on hydration kinetics of sesame seeds (Sesamum indicum L.), doi:10.1016/j.jfoodeng.2008.10.010, J Food Eng, 91 (4), 542-552 (2009)

Krokida, M. K., y C. Philippopoulos, Rehydration of dehydrated foods, doi: 10.1081/DRT-200054201, Dry Technol, 23, 799-830 (2005)

Lucas, T., D. Le Ray, y F. Mariette, Kinetics of water absorption and solute leaching during soaking of breakfast cereals, doi:10.1016/j.jfoodeng.2005.11.006, J Food Eng, 80, 377-384 (2007)

Marin, B., M. Lemus, M. Flores, y G. Vega, La rehidratación de alimentos deshidratados, doi: org/10.4067/S0717-75182006000500009, Rev Chil Nutr, 33 (3), 527-538 (2006)

Melquíades, Y. I., C. López, M. E. Rosas, Estudio de la cinética de rehidratación de zanahoria (Daucus Carota) deshidratadas, doi:10.1612/inf.tecnol.4037it.08, Inf Tecnol, 20(3), 65-72 (2009)

Muñoz, I., N. Garcia-Gil, J. Arnau, y P. Gou, Rehydration kinetics at 5 and $15{ }^{\circ} \mathrm{C}$ of dry salted meat, doi:10.1016/j.jfoodeng.2011.12.020, J Food Eng, 110, 465-471 (2012)

Paksoy, M., y C. Aydin, Determination of some physical and mechanical properties of pea (Pisum sativum L.) seeds, doi: 10.3923/pjbs.2006.26.29, Pak J Biol Sci, 9 (1), 26-29 (2006)

Parthasarathi, S., y C. Anandharamakrishna, Modeling of shrinkage, rehydration and textural changes for food structural analysis: a review, doi:10.1111/jfpe.12073, J Food Process Eng, 37, 199-210 (2014)

Salimi, A., Y. Maghsoudlou, y S. M. Jafari, Effect of water temperature, variety and shelf life on rehydration kinetics of microwave dried potato cubes, http://www.scielo.org.ar/scielo.php?script=sci_arttext\&pid=S032707932011000300008\&Ing=es\&nrm=iso>; ISSN 0327-0793, Lat Am Appl Res, 4, 249-254 (2011)

Shafaei, S. M., y A. A. Masoumi, Evaluation of khazaei model in predicting of water absorption of chickpea during soaking, doi:10.14196/aa.v3i1.1101, Agric Adv, 3(1), 1-8 (2014a)

Shafaei, S. M., y A. A. Masoumi, Estimating moisture absorption kinetics of beans during soaking using mathematical models, http://www.cigrjournal.org, Agric Eng Int, 16 (3), 230-237 (2014b)

Sharma, V., L. Das, R. C. Pradhan, S. N. Naik, N. Bhatnagar, y R. S. Kureel. Physical properties of tung seed: an industrial oil yielding crop, doi:10.1016/j.indcrop.2010.10.031, Ind Crops Prod, 33(2), 440-444 (2011)

Sopade, P., P. Yu Xun, P. Halley, y M. Hardin, Equivalence of the Peleg, Pilosof and Singh-Kulshrestha models for water adsorption in food, doi:10.1016/j.jfoodeng.2005.10.007, J Food Eng, 78(2), 730-734 (2007) 
Szymanowska, U., A. Jakubczyk, B. Baraniak, y A. Kur, Characterisation of lipoxygenase from pea seeds (Pisum sativum var. Telephone L.), Food Chem, 116, 906-910 (2009)

Téllez-Pérez, C., M. M. Sabah, J. G. Montejano-Gaitán, V. Sobolik, C. A. Martínez, y K. Allaf, Impact of instant controlled pressure drop treatment on dehydration and rehydration kinetics of green Moroccan pepper (Capsicum annuum), doi:10.1016/j.proeng.2012.07.491, Procedia Eng, 42, 978-1003 (2012)

Turhan, M., S. Sayar, S. Gunasekaran, Application of Peleg model to study water absorption in chickpea during soaking, doi:10.1016/S0260-8774(01)00152-2, J Food Eng, 53 (2), 153-159 (2002)

Villalobos, M., A. Patel, V. Orsat, J. Singh, y M. Lefsrud, Fatty acid profiling of the seed oils of some varieties of field peas (Pisum sativum) by RP-LC/ESI-MS/MS: Towards the development of an oilseed pea, doi:10.1016/j.foodchem.2012.12.052, Food Chem,139 (1-4), 986-993 (2013)

Wang, N., D. W. Hatcher, E. J. Gawalko, Effect of variety and processing on nutrients and certain antinutrients in field peas (Pisum sativum), doi:10.1016/j.foodchem.2008.03.047, Food Chem, 111,132-138 (2008)

Wang, S., P. Sharp, y L. Copeland, Structural and functional properties of starches from field peas, doi:10.1016/j.foodchem.2010.11.154, Food Chem, 126, 1546-1552 (2011)

Yalcin, I., C. Ozarslan, y T Akba, Physical properties of pea (Pisum sativum) seed, doi:10.1016/j.jfoodeng.2006.02.039, J Food Eng, 79(2), 731-735 (2007)

Yildirim, A., M. D. Öner, y M. Bayram, Fitting Fick's model to analyze water diffusion into chickpeas during soaking with ultrasound treatment, doi:10.1016/j.jfoodeng.2010.12.005, J Food Eng, 104, 134-142 (2011)

Yildirim, A., M. D. Öner, y M. Bayram, Modeling of Water Absorption of Ultrasound Applied Chickpeas (Cicer arietinum L.) Using Peleg's Equation, http://dergiler.ankara.edu.tr/dergiler/15/1726/18350.pdf, Tarım Bilim Derg - J Agric Sci, 16, 278-286 (2010) 
